\title{
Clear Cell Hidradenocarcinoma: a Rare Identity Difficult to Recognize - Case Report and Review of the Literature
}

\author{
Danilo COCO ${ }^{a}$, Silvana LEANZA ${ }^{b}$, Roberto CAMPAGNACCI ${ }^{b}$ \\ aDepartment of General Surgery, Ospedali Riuniti Marche Nord, Pesaro (PU), Italy \\ bepartment of General Surgery, Carlo Urbani Hospital, Jesi (AN), Italy
}

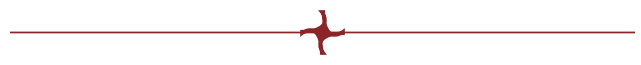

\begin{abstract}
Hidradenocarcinoma (HC) is a malignant and aggressive sweat gland tumour. It is a rare occurrence, accounting for about $6 \%$ of malignant eccrine cancers and less than $1 \%$ of all cutaneous neoplasms. It is a malignant tumor which can manifest itself in any area of the body, including the anterior surface of the trunk, the torso, scalp, face, and extremities. It is apparent that HC has a high propensity for lymphatic metastasis. The suggested treatment approach for HC implies making a wide surgical excision with negative margins. Nevertheless, such tumours are aggressive and recur locally in 50\% of cases; they also produce metastases in $60 \%$ of cases. The overall five-year survival rate is 30\%. Here, we present the case of a 68-year-old Caucasian male with unknown diagnosis of malignant neoplasm of inguinal cutaneous skin of sweat gland called hydrocarcinoma.
\end{abstract}

Keywords: hidradenocarcinoma, clear cell hidradenoma, malignant cell hidradenoma, spiradenoma, surgery.

\section{INTRODUCTION}

T The term "hidradenoma" was initially reported by Mayer in 1941, whereas in 1954, Keasbey and Hadley suggested the name "clear cell hidradenoma" (1). Hidradenocarcinoma $(\mathrm{HC})$ is a malignant and aggressive sweat gland tumour with a rare occurrence of about $6 \%$ of malignant eccrine cancers that account for $0.001 \%$ of all tu- mours (2-4). Among females, the main occurrence of this disease is among those aged $50-70$ years $(5,6)$. This condition appears as either cystic or solid subcutaneous nodules that are linked to ulceration or pruritus (2). The most frequently involved areas are the scalp (among 40\% of all patients) and face (30\%), while the extremities are only rarely affected.

The suggested treatment approach for $\mathrm{HC}$ implies making a wide surgical excision with nega-

\footnotetext{
Address for correspondence:

Danilo Coco, MD

Mailing address: Ospedali Riuniti marche Nord, Pesaro (PU), Italy

Email: webcostruction@msn.com
} 
tive margins $(4,7)$. However, after surgical resection the recurrence rate of this disease ranges between $10-50 \%(4,5,8)$. A possible means of detecting lymph node metastasis is through sentinel lymph node biopsy (SLNB) and preoperative lymphatic mapping (6). It is apparent that $\mathrm{HC}$ has a high propensity for lymphatic metastasis in case of less than $2 \mathrm{~mm}$ invasion as well as poor differentiation and perineural invasion ( $R$. Delgado) (6). Furthermore, radiotherapy (RT) and chemotherapy $(\mathrm{CHT})$ are regarded as the gold standard $(\mathrm{CHT}+\mathrm{RT})(4,9)$. The regimen consisting of 5-Fluorouracil (5-FU) plus capecitabine seems to be the first line treatment while cyclophosphamide, doxorubicin, platins or vincristine, with bleomycin being the second line thrapy (10). Moreover, trastuzumab was used in order to stabilise disease progression in a tumour with HER2/neu expression (10).

In this paper, we describe a case report regarding a 68-year-old Caucasian male with a histological finding of hidradenoma in his right skin inguinal region, which subsequently showed a previous unknown clear cell hidradenocarcinoma.

\section{CASE REPORT}

$\mathrm{O}$ ur patient was a 68-year-old Caucasian male with a history of rectal resection for cancer and previous excision of left inguinal skin benign neoplasm. Histology of the skin indicated that he had an eccrine spiradenoma of the sweat glands together with intense desmoplastic reaction and squamous differentiation, a so-called hidroadenoma or benign clear cell adenoma. Moreover, the patient was assessed in 2020 for a new rapidly growing ulcerous lesion and a septal appearance on the skin in the same inguinal area. A similar histology was confirmed after the second excision as well as after three further consecutive skin resections for recurrence. During the subsequent six months after the last resection, the lesion and septal appearance on the skin of this area and also multiple satellite nodules were compared again.

In order to receive additional diagnosis and definitive treatment, the patient was admitted to our institution when the inguinal mass re-appeared. His medical history included essential arterial hypertension, a history of rectal resection for cancer and previous and multiple excision of this left inguinal skin as benign neoplasm. During hospitalization, the patient had a good general condition, with good performance status and good nutritional values. His temperature was $36^{\circ} \mathrm{C}$, with blood pressure of $130 / 62 \mathrm{mmHg}$, heart rate of 66 regular beats/min, and respiratory rate of 15 breaths/min. Laboratory testing revealed hemoglobin of $10.8 \mathrm{~g} / \mathrm{dL}$, platelet count

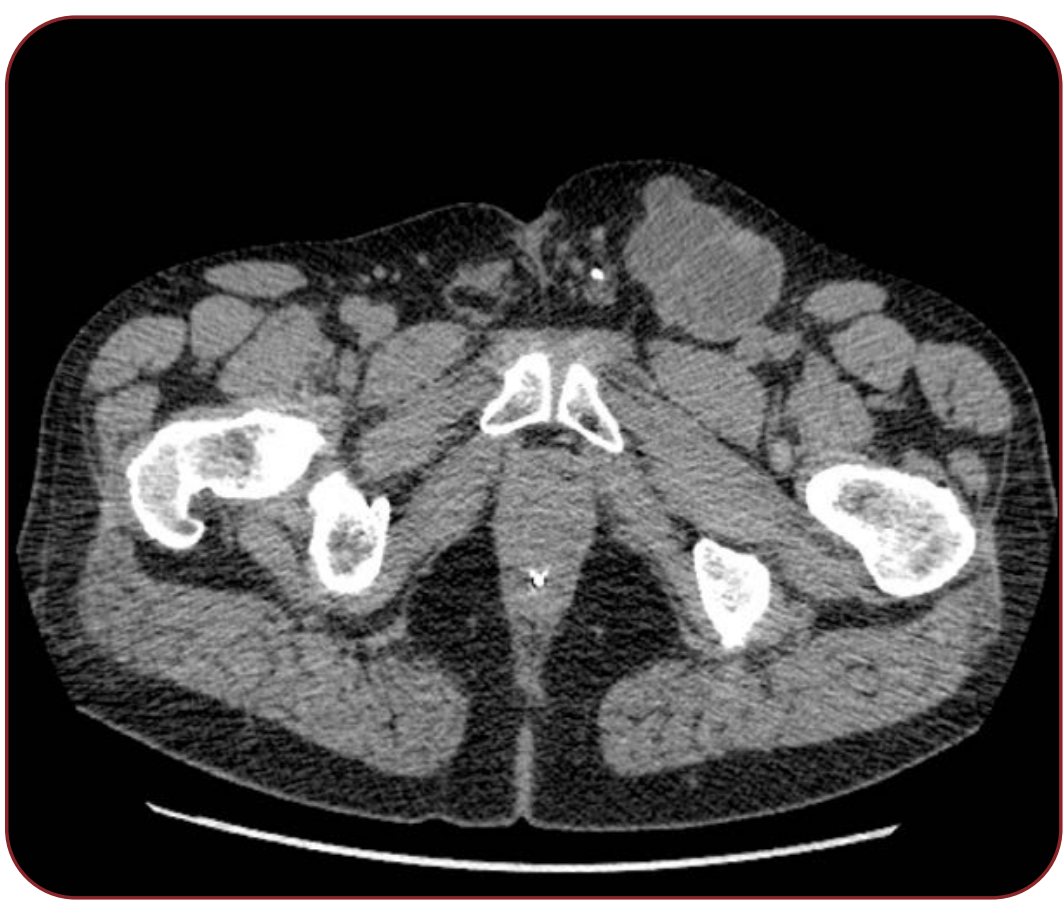

F I GURE 1. Computed tomography followed by magnetic resonance imaging confirming the presence of the mass and showing ipsilateral iliac-femoral lymphadenopathy 
of $120,000 / \mathrm{mm}^{3}$, increased serum urea of $0.60 \mathrm{~g} / \mathrm{dL}$ and serum creatinine of $1.7 \mathrm{mg} / \mathrm{dL}$, with an elevated white blood cell (WBC) count of $19,300 / \mu \mathrm{L}$ and C-reactive protein (CRP) of $100.2 \mathrm{mg} / \mathrm{dL}$. Physical examination of the abdomen was unremarkable. In the inguino-scrotal region, a mass of about four square meters in diameter which was fixed on the underlying anatomical plans, ulcerated and with regular margins, has been found. A computed tomography (CT) followed by magnetic resonance imaging (MRI) confirmed the presence of the mass and identified ipsilateral iliac-femoral lymphadenopathy (Figure 1). Furthermore, positron emission tomography (PET) verified the presence of the lesion and revealed a lymph node contrast enhancement of ipsilateral iliac-femoral vessels, whereas for ipsilateral inguinal nodal disease, lymphoscintigraphy was positive.

Moreover, an iliac-femoral lymph node dissection and a funicolo-orchiectomy were conducted. Additionally, a wide excision of the abdominal wall nodules, with $2 \mathrm{~cm}$ resection margins, was performed. Histologic finding of the inguinal skin showed anastomising cords and lobulated masses of cells with a polygonal to slightly fusiform shape, cells appearing as bland ovoid nuclei with clear cytoplasm. Focal areas within the lesion may indicate the presence of malignant changes, large pleomorphic hyperchromatic nuclei, with a tendency towards squamous differentiation with numerous neoplastic cells (Figure $2 \mathrm{a}, \mathrm{b}, \mathrm{c}, \mathrm{d}$ ). The histologic finding of lymph node revealed metastasis of carcinoma, comprising cells with a clear, abundant glycogen-rich cytoplasm and moderate to severe nuclear types. Furthermore, lumens which contained PASd + material were emphasized by IHC staining for CEA. Given that the IHC test indicated positivity for p63, EMA and CAIX, previous slides were re-investigated as they had shown clear cell hidradenoma. However, since every previous slide was superimposable with the last one, the same diagnosis was also confirmed for the three prior excisions of the lesion, confirming clear cell hidradenocarcinoma and lymph node metastases. This has also showed that the tumour had invaded the subcutaneous fat and the dermal lymphatic vessels, but the resection margins had not been affected by the disease. Moreover, a clear cell hidradenocarcinoma emanating from the sweat glands with multiple nodal metastases was highlighted by the pathology report. Adjuvant treatment was not provided. The patient was discharged from the hospital on the third day after admission. He was alive at one year after the last excision.

\section{DISCUSSION}

lear-cell hidradenocarcinomas account for no more that $6 \%$ of malignant eccrine gland tumours and below $1 \%$ of all cutaneous neoplasms. These include the anterior surface of the trunk, the torso, the scalp, the face, and extremities; however, only 50 cases are reported in the literature (11). This condition occurs most fre-

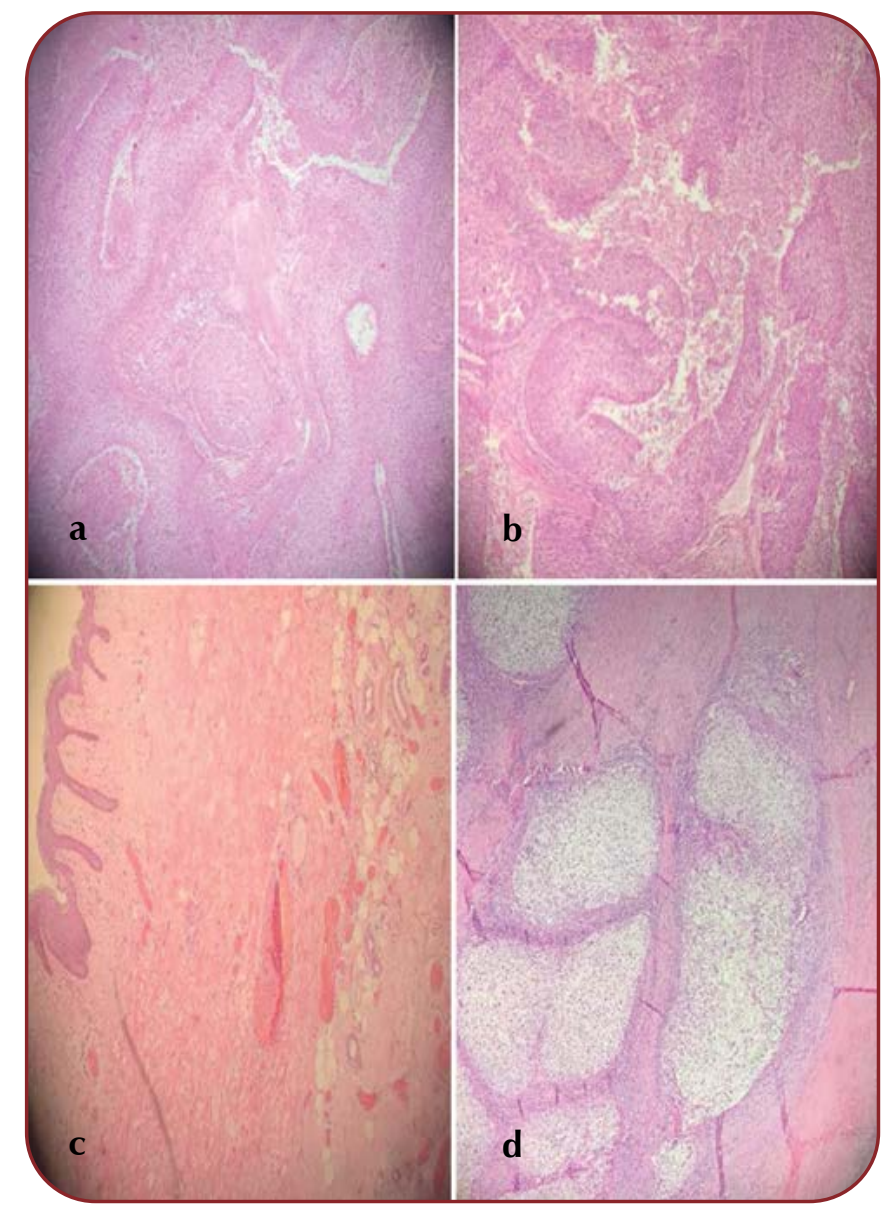

F I GURE 2 a, b, c, d. Histological sections indicating anastomising cords and lobulated masses of cells with a polygonal to slightly fusiform shape, whereas most cells appear as bland ovoid nuclei with slightly eosinophilic to clear cytoplasm. Focal areas within the lesion may demonstrate malignant changes, and possibly show several mitotic figures, large pleomorphic hyperchromatic nuclei, with a tendency towards squamous differentiation with numerous neoplastic cells indicating individual keratinisation. 
quently in women, especially among those in the fifth decade of their lives (12). This is a slowly-growing lesion over several years and takes the form of a painless and firm subcutaneous nodule which has the potential to secrete serosanguinous fluid. Nevertheless, such tumours are aggressive and recur locally in $50 \%$ of cases; they also produce metastases in the brain, bone, lung, liver as well as the mediastinal or peritoneal lymph nodes in up to $60 \%$ of cases in a two-year period. Furthermore, according to Souvatzidis et al (13), the overall five-year survival rate is $30 \%$. In instances of the primary disease and local recurrences, Wong et al (14) recommended wide surgical resection of a minimum of $2 \mathrm{~cm}$ clear margins. Histological sections indicate anastomising cords and lobulated masses of cells with a polygonal to slightly fusiform shape, whereas most cells appear as bland ovoid nuclei with slightly eosinophilic to clear cytoplasm. Focal areas within the lesion may demonstrate malignant changes, and possibly show several mitotic figures, large pleomorphic hyperchromatic nuclei; they also reveal a tendency towards squamous differentiation with numerous neoplastic cells indicating individual keratinisation (15). It appears that clear cell hidradenoma emanates from the ductal epithelium of the sweat glands. However, histogenetically, it seems to represent a transitional tumour which has features in common with eccrine spiradenoma and eccrine poroma (16). Clear cell hidradenoma comprises two principal cell subpopulations: the first one consists of round or polygonic cells with round nuclei and clear cytoplasm caused by abundant glycogen storage, and the second one comprises multifaceted cells of asophilic cytoplasm and oval nuclei evenly arranged at the periphery of the first cellular line (17). Extensive surgical resection of the tumour (3 to $5 \mathrm{~cm}$ margins being recommended to gua- rantee negativity) is the only treatment that has positively influenced survival. Diagnostic surgery is usually undertaken - a second intervention is needed to study the lymph nodes and extend the margins after having established the tumour type. Sentinel lymph nodes should be studied and, if their involvement is confirmed, lymphadenectomy connected with radiotherapy is necessary $(10,18)$. However, it has been demonstrated that adjuvant treatment applying radiotherapy and chemotherapy has no effect on either survival or local control of the disease (19). Recurrences are treated according to reintervention, with or without related radiotherapy, also assessing the likelihood of directed treatment or targeted therapy against HER2 (Herceptin1) (8). Interestingly, that case report describes a rare identity which is difficult to recognize. In our case, continuous excision for local recurrence of inguinal neoformations showed a clear cell hidradenocarcinoma rather than hidroadenoma. $\square$

\section{CONCLUSION}

abbaridia et al (20) state that, although HC is La rare eccrine gland tumour, it is nevertheless an aggressive one, and there are no current evidence-based guidelines. However, using sentinel lymph node biopsy, radiotherapy (RT) and chemotherapy (CHT) is still a controversial topic in HC cases (21). As shown by the present report, hidradenoma is a benign tumour, which is nevertheless difficult to differentiate from hidradenocarcinoma. We suggest a complete surgical resection with wide margins, even where no cytological malignancy exists.

Conflicts of interest: none declared.

Financial support: none declared.

Acknowledgments: Danilo Coco, the corresponding author of this article, and Silvana Leanza, MD, co-author.

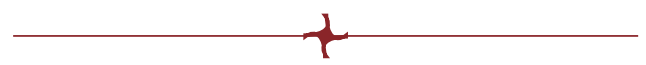

\section{R}

1. Keasby LE, Hadley GG. Clear cell hidradenoma. Cancer 1954;7:934-951.

2. Avraham JB, Villines D, Maker VK, et al. Survival after resection of cutaneous adnexal carcinomas with eccrine differentiation: risk factors and trends in outcomes.

J Surg Oncol 2013;108:57-62.

3. Moore RS. Report of case.

The Laryngoscope 1923;33:212-212.

4. Tolkachjov SN, Hocker TL,
Hochwalt PC, et al. Mohs micrographic surgery for the treatment of hidradenocarcinoma: the mayo clinic experience from 1993 to 2013. Dermatologic Surgery 2015;41:226-231.

5. Yavel R, Hinshaw M, Rao V, et al. 
Hidradenomas and a

hidradenocarcinoma of the scalp managed using Mohs micrographic surgery and a multidisciplinary approach: case reports and review of the literature.

Dermatologic Surgery 2009;35:273-281.

6. Delgado R, Kraus D, Coit DG, Busam KJ. Sentinel lymph node analysis in patients with sweat gland carcinoma. Cancer 2003;97:2279-2284.

7. Giannelli F, Chiola I, Belgioia L, et al. Complete response in a patient with gynaecological hidradenocarcinoma treated with exclusive external beam radiotherapy and brachytherapy: a case report. Journal of Contemporary Brachytherapy 2017;9:572-578.

8. Amel T, Olfa G, Faten H, et al.

Metastatic hidradenocarcinoma: surgery and chemotherapy.

North American Journal of Medical Sciences 2009;1:372-374.

9. Khan MB, Mansha MA, Ali N, et al. Hidradenocarcinoma: five years of local and systemic control of a rare sweat gland neoplasm with nodal metastasis.
Cureus 2018;10:e2884.

10. Gauerke S, Driscoll JJ. Hidradenocarcinoma: a brief review and future directions. Arch Pathol Lab Med 2010;134:781-785.

11. Sehgal S, Goyal P, Ghosh S, et al. Clear cell hidradenoma of breast mimicking atypical breast lesion: a diagnostic pitfall in breast cytology. Rare Tumors 2014;6:5343.

12. Liapakis IE, Korkolis DP, Koutsoumbi A, et al. Malignant hidradenoma: a report of two cases and review of the literature. Anticancer Res 2006;26:2217-2220.

13. Souvatzidis $P$, Sbano $P$, Mandato F, et al. Malignant nodular hidradenoma of the skin: report of seven cases.

J Eur Acad Dermatol Venereol 2008;22:549-554.

14. Wong TY, Suster S, Nogita D, et al. Clear cell eccrine carcinomas of the skin. $\infty$ clinicopathologic study of nine patients. Cancer 1994;73:1631-1643.

15. Stromberg BV, Thorne IS Dimino-Emme L, et al. Malignant clear cell hidradenoma: a case report and literature review.
Nebr Med J 1991;76:166-170.

16. Lever WF, Schaumburg-Lever G. Histopathology of the Skin, $6^{\text {th }}$ Edition, JB Lippincot, Philadelphia, 1983, pp 557-585.

17. Hashimofo F, Di Bella RJ, Lever WF. Clear cell hidradenoma. Arch Dermatol 1967;96:18-38.

18. Mote, D.G., Ramamurti, $T$, Naveen, Babu B. Nodular hidradenoma of the breast: a case report with literature review.

Indian J Surg 2009;71:43-45.

19. Soni A, Bansal N, Kaushal V, Chauhan AK. Current management approach to hidradenocarcinoma: a comprehensive review of the literature. Ecancermedicalscience 2015;9:517.

20. Labbardia W, Marnissib F, Cribierc B, Chiheba S.

Hidradenocarcinoma of the heel associated with inguinal metastases. Annales de Dermatologie et de Vénéréologie 2017;144:536-542.

21. Rafols M, Mejia O, Oh KS, et al. An Unusual Case of Lower Extremity Clear Cell Hidradenocarcinoma. Case Rep Surg 2020:6192109. https://doi.org/10.1155/2020/6192109 